\title{
Square pulse heating infrared thermography and shearography applied simultaneously on CFRP tissue bonded to reinforced concrete
}

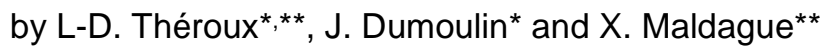 \\ * LUNAM Université, IFSTTAR, COSYS/SII, Route de Bouaye, CS4, F-44340, Bouguenais, France \\ ** Laval University, Department of Electrical and Computer Engineering, LVSN/MIVIM, Av. De la Médecine, \\ Québec (Québec), Canada
}

\begin{abstract}
This study concern the use of a common thermal excitation for infrared thermography and shearographic measurements. The two NDT methods are used simultaneously to detect defects in the bonding between a concrete slab and a tissue of carbon fiber reinforced polymer (CFRP). Experimentation was conducted to evaluate defects depending on their size and simulated bonding quality. A numerical model was designed according to the experimental conditions. Obtained simulated results were compared with experimental data that allowed the validation of the numerical model. Analysis of the results shed some light on the relation between the thermal and mechanical response on defective samples.
\end{abstract}

\section{Context}

The aging of concrete structure as long been a concern in civil engineering. Over the lifetime of the structure, atmospheric exposure, traffic loading and application of de-icing salts affect their resilience [1]. As they age, structures integrity diminish with time which is why different retrofitting methods are required. Among those, the application of carbon fiber reinforced polymer (CFRP) was proven as an effective method to reinforce failing concrete structure [2]. The efficiency of such reinforcement depends greatly on the bonding quality. So, the installation of this reinforcement system require technician to follow rigorous step to make sure the bonding of the CFRP on the surface is strong enough for the reinforcement to be effective. Nevertheless, there may be flaws in the gluing. Delaminations, cracks and other cavities that cause irregularities in the surface may create small pockets of air in the bonding layer. Furthermore, the presence of volatile contaminant or humidity may reduce the effectiveness of the gluing. The goal of this paper is to determine how the quality of the bonding can be evaluated. Many non-destructive testing (NDT) have been developed and studied in this regards. Infrared thermography is one of those well-known methods. Past researches have shown that it could successfully detect, and in some extent even characterize defects in the bonding [3-7]. However, the presence of flaws in the bonding does not necessarily mean the bonding is entirely compromised. The bonding quality would be better evaluated by a testing method that mostly depends on the mechanical properties of the experimental subject. For example, pull-off adhesion tests are a reliable method to give a value of the bonding strength [8]. However, this method has a limited area of inspection and destroys the portion of surface inspected. The next possible solution is thermal shearography that has the advantage to be a NDT that is contactless and full-field [9]. This study investigates the coupling of thermal shearography coupled with thermography.

\section{Shearography}

Shearography gives the relative out-of-plane displacement of a surface. To do this, it uses holography to measure the phase map of the surface of the inspected subject. The base principle of holography is to project a coherent light beam on the surface and make the reflected beam interfere with a reference beam emitted by the same source. The resulting interference, either constructive or destructive, gives the phase map. This phase map doesn't yield much information because it is noised by the small irregularities in the structure surface. To make the most out of a phase map, it must be compared to another phase map. The procedure is the following: a reference phase map is first taken when the subject is unstressed. Afterwards, displacements are induced by applying some form of stress in the structure (thermal stress in our case). By comparing this phase map to the reference phase map, it is possible to calculate the outof-plane displacement. When the surface is deformed, it changes the value of the optical path length (OPL). In fact, it changes that value by a factor of two since the beam travel back and forth. Taking into account that the point of observation and the illumination source share the same position and are both perpendiculars to the surface, we have [10]:

$$
\triangle O P L=2 w
$$

Where $w$ is the out-of-plane displacement. 
Since we know that a complete phase cycle correspond to $2 \pi$ for a distance of one wavelength $\lambda$, we obtain the following equation:

$$
\Delta \varphi=\frac{2 \pi}{\lambda} \cdot 2 w
$$

Practical uses of holography have shown that such an experimental setup is very susceptible to noise. Vibrations and even small change in the air refractive index can invalidate the phase measurements. To solve this problem, both the reference and the reflected beam must share a similar path. By doing this, vibrations affect the OPL of both beams equally. To achieve this, the beam reflected on the object is focussed in a Michelson interferometer. By tilting one of the mirrors, the object beam, instead of interfering with the reference beam, will interfere with a sheared version of itself (consequently the name shearography). The relation between the phase difference $\Delta \varphi$ and the displacement become the following:

$$
\Delta \varphi=\frac{2 \pi}{\lambda} \cdot 2\left(\frac{\partial w}{\partial x}\right) \delta_{x}
$$

With $\delta_{x}$ the shearing distance induced by the tilted mirror, in other word, the offset between the two images created when tilting the mirror in the Michelson interferometer. The shearing direction in the axis $x$ was taken arbitrarily and can be modified by changing the tilt orientation of the mirror. Finally, the camera cannot directly measure the phase map, it can only measure the light intensity that can be described by the subsequent equation [11]:

$$
I_{N}=I_{0}\left(1+m \cdot \cos \left(\varphi+\varphi_{N}\right)\right)
$$

Where $I_{0}$ is the normalized intensity, $m$ is the contrast and $\varphi$ the phase we want to calculate. Since there are three unknown variables, we need at a minimum three equations to solve this system. Each equation correspond to one image, between each acquisition a dephasing $\varphi_{N}$ must be induced by moving one of the mirror in the Michelson interferometer The dephasing increment is $2 \pi / N$ where $N$ is the number of images for the phase extraction. In this study, it was decided to use a phase extraction using four images. The relation between each image and the phase becomes:

$$
\varphi=\frac{\arctan \left(I_{4}-I_{2}\right)}{I_{1}-I_{3}}
$$

Other methods for phase extraction are also available, such as using 3 or 5 images, as well as using a continuous phase extraction. When making shearohgraphic measurement, it may happen that the curl of displacement is important enough that the phase difference is higher than a half-wavelength. This will cause fringe to appear and the phase must then be unwrapped to make the transition of $2 \pi$ continuous. The obtained image is the value of the curl of displacement. This image must be uncurled to have the raw value of out-of-plane displacement. More information can be found in the PhD thesis of F. Taillade [12].

\section{Experimentation}

To test the feasibility has well has the limitations and efficiency of practical thermal shearography coupled with infrared thermography, a series of experiments were conducted as part of this study. Two different samples were tested, both made of a concrete slab with CFRP tissue glued by impregnating bi-component epoxide thermosetting resin. To simulate the presence of defect in the bonding, discs made of polytetrafluoroethylene (PTFE) were inserted between the concrete and the tissue. The first sample uses defect of various size while the second sample uses defect with different bonding quality. PTFE was used instead of other material because it simulates the thermal behaviour of air. The thermal conductivity of air is very low (roughly $0.025 \mathrm{~W} / \mathrm{mK}$ ) and we know for a fact that PTFE does not chemically bond with the epoxy glue, creating a thermal contact resistance that decrease PTFE effective thermal conduction coefficient.

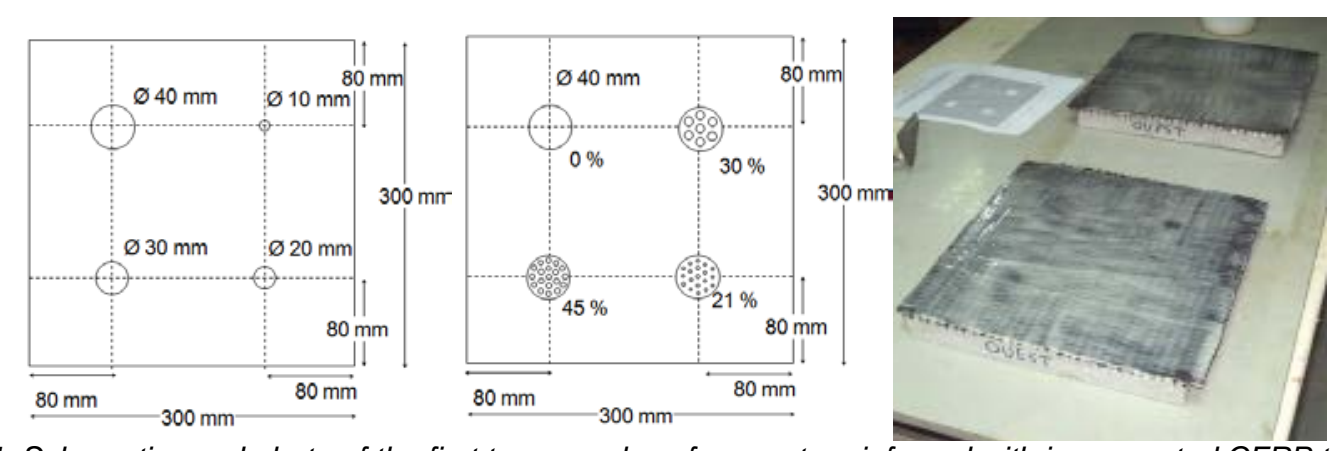

Fig. 1. Schematics and photo of the first two samples of concrete reinforced with impregnated CFRP tissue. The first sample contain defect of various size while the other have disc with holes inside to simulate different bonding quality. 
One of the major challenges, when coupling thermography and shearography, concern the choice of the heating source. The initial setup involved only thermography and the heat excitation was made by two halogen lamps that radiate in the visible spectrum (see [7]). The coherent length source used had a wavelength in the visible spectrum. So using halogen lamp during a shearographic measurements will affect the efficiency of the system. To solve this problem, carbon lamps were used. The particularity of those type of lamp is that they radiate mostly in the infrared band and do not hamper the shearographic acquisition.

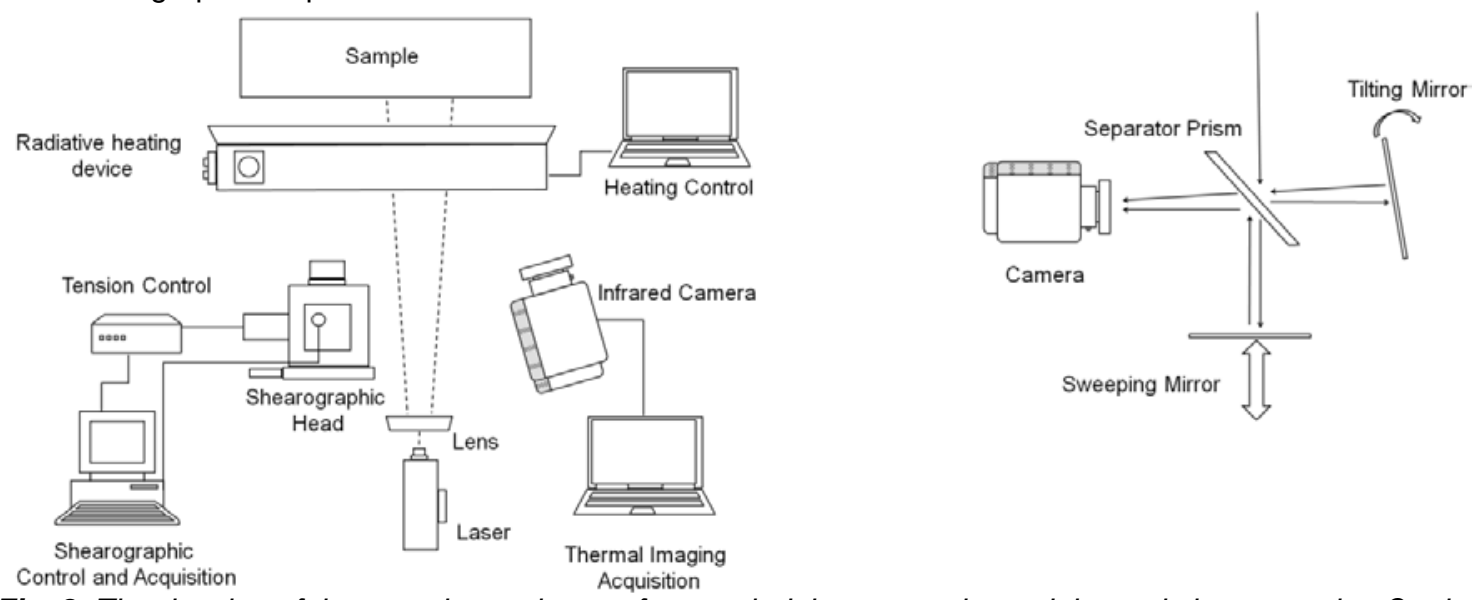

Fig. 2. The drawing of the experimental setup for coupled thermography and thermal shearography. On the right, the detailed schematic of the shearographic head.

The heating source and each acquisition system were controlled with different hardware and dedicated software. As for the in-house shearographic system developed by F. Taillade (see [9]) no synchronisation was possible. A post synchronisation was realized by using timestamp information available with corresponding measured data stored on each hardware. For the shearographic measurements, the coherent light source used was a Nd:YAG laser with a wavelength of $532 \mathrm{~nm}$. The infrared measurement were carried out with a FLIR SC655 uncooled microbolometric IRFPA camera of $640 \times 480$ pixels in the LWIR bandwidth.

\subsection{Shearographic Measurement Results}

For the two samples, the images from the $40 \mathrm{~mm}$ wide defects are the most promising. For the other discs, the detectability by direct visual analysis of the phase images decrease with size of the defects. Lower bonding quality defective areas yield also to same observation. In figure 3, the first three phase images were taken during the heating while the last three were taken during the relaxation.
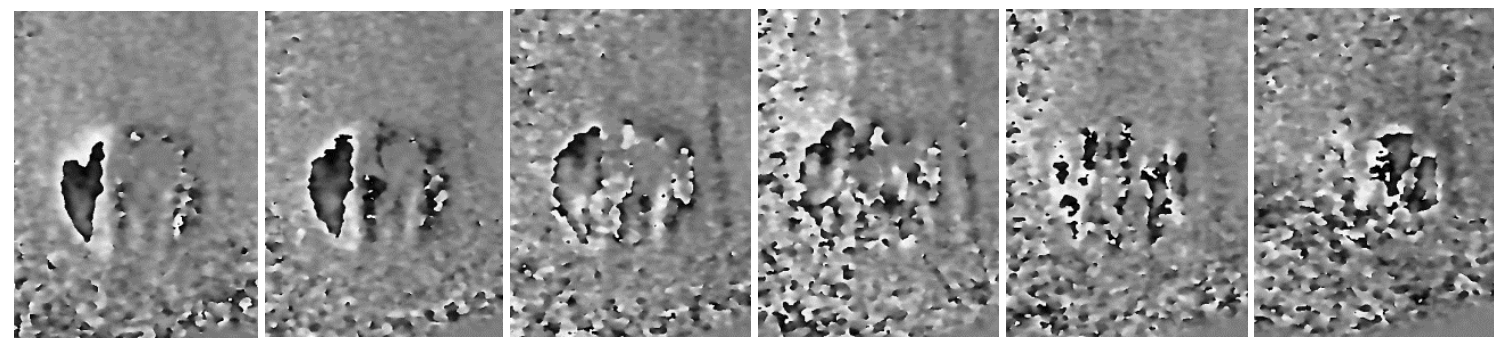

Fig. 3 The shearographic images of the $40 \mathrm{~mm}$ wide defect. From the left to the right, the first 3 image were taken during the heating process over a period of 3 minutes.

\subsection{Infrared Measurement Results}

The results from active infrared thermography show that we were able to locate all the defects (see Figure 4). In general, the visibility was clearer than for images obtained by shearography. 


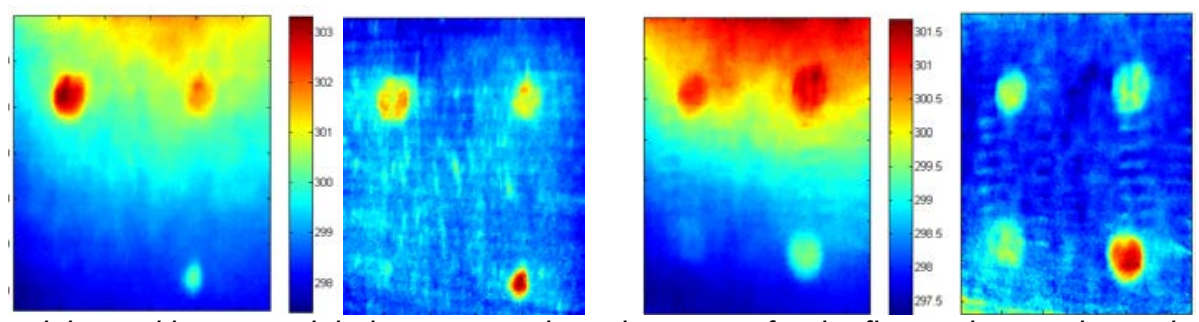

Fig. 4. Infrared thermal image and their correspondent phase map for the first and second sample respectively on the left and on the right.

Even if the heating was not evenly distributed, the phase map calculated gives a good indication of the size and shape of the defects.

\section{Numerical simulations}

To have a better understanding of the experimental results in regard of the thermomechanical behavior of the sample, numerical models were conceived under $\mathrm{COMSOL}^{\odot}$. These models are based on the real-life samples and try to recreate the same experimental condition. For the heat transfer conditions; the top surface of the model is subjected to a uniform heat flux of $2 \mathrm{~kW} / \mathrm{m}^{2}$ conditioned by a square pulse with a duration of 30 seconds. The edges of the PTFE discs were designed as a thin thermally resistance of air to simulate the thermal resistance contact. The initial temperature considered was set at the ambient temperature value of $293 \mathrm{~K}$. The exterior surfaces of the model are also subject to convection cooling with a coefficient of $10 \mathrm{~W} / \mathrm{m}^{2} \mathrm{~K}$.

For the structural mechanic condition, for all the components, the behavior is considered as linearly elastic. This approximation is valid since the deformations involved in the simulation are infinitesimal. Of course, the model is subjected to thermal expansion with the reference temperature taken from the solver of the heat transfer module calculation. The bottom of the samples is fixed since in the experiment it was laying on a table. The edges of the defects disc are free since PTFE disc do not chemically bond the epoxy glue. The materials properties used in the model are reported in Tables 1 and 2.

Table 1. Materials properties used in the numerical models

\begin{tabular}{llccccc}
\hline Properties & & Concrete & Époxy & Composite & PTFE & Air \\
\hline Thermal Conductivity & {$\left[\mathrm{W} /\left(\mathrm{m}^{\star} \mathrm{K}\right)\right]$} & 1,8 & 0,2 & $4,2 / 0,7 / 0,7$ & 0,235 & 0,024 \\
Heat Capacity & {$\left[\mathrm{J} /\left(\mathrm{kg}^{\star} \mathrm{K}\right)\right]$} & 920 & 1220 & 840 & 1050 & 1006 \\
Density & {$\left[\mathrm{kg} / \mathrm{m}^{\wedge} 3\right]$} & 1200 & 1200 & 1530 & 2200 & 1,225 \\
Young's Modulus & {$[\mathrm{GPa}]$} & 30 & 10,5 & Ref. Table 2 & 0,5 & - \\
Thermal Expansion Coeff. & {$[1 / \mathrm{K}]$} & $1,20 \mathrm{E}-05$ & $2,50 \mathrm{E}-05$ & $-0,8 / 35 / 35 \mathrm{E}-06$ & $1,35 \mathrm{E}-04$ & - \\
Poisson's Ratio & - & 0,21 & 0,4 & 0,33 & 0,46 & - \\
\hline
\end{tabular}

Table 2. Rigidity tensor of the carbon/epoxy laminate T300/914 (see [9])

\begin{tabular}{cccccc}
\hline 143,8 & 6,2 & 6,2 & 0 & 0 & 0 \\
6,2 & 13,3 & 6,5 & 0 & 0 & 0 \\
6,2 & 6,5 & 13,3 & 0 & 0 & 0 \\
0 & 0 & 0 & 3,6 & 0 & 0 \\
0 & 0 & 0 & 0 & 3,6 & 0 \\
0 & 0 & 0 & 0 & 0 & 5,7 \\
\hline
\end{tabular}

The time step used during numerical simulations was set to $1 \mathrm{~s}$ during $300 \mathrm{~s}$.

\subsection{Results}

In figure 5 an illustration of simulated surface temperature distribution for the two studied samples is presented. Such thermal maps can also be used to generate simulated thermal image sequence in order to test the robustness of the processing algorithms studied versus measurement noise. 

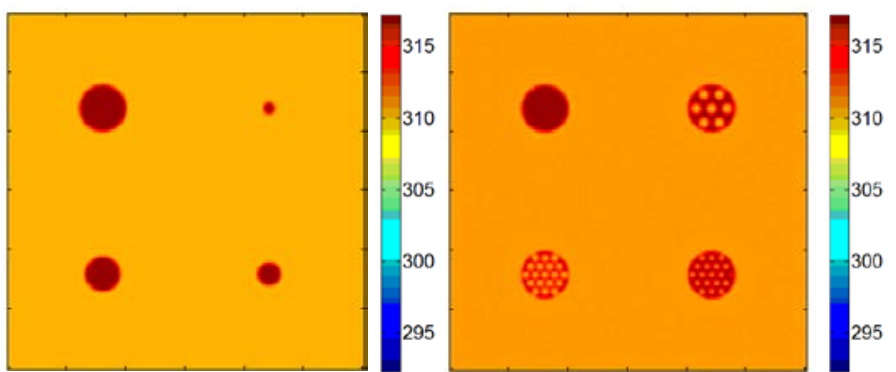

Fig. 5. Simulated infrared thermal image of the first and second sample. The images are taken at the end of the heating.

In figure 6 an illustration of simulated surface displacement distribution for the two studied samples is presented.
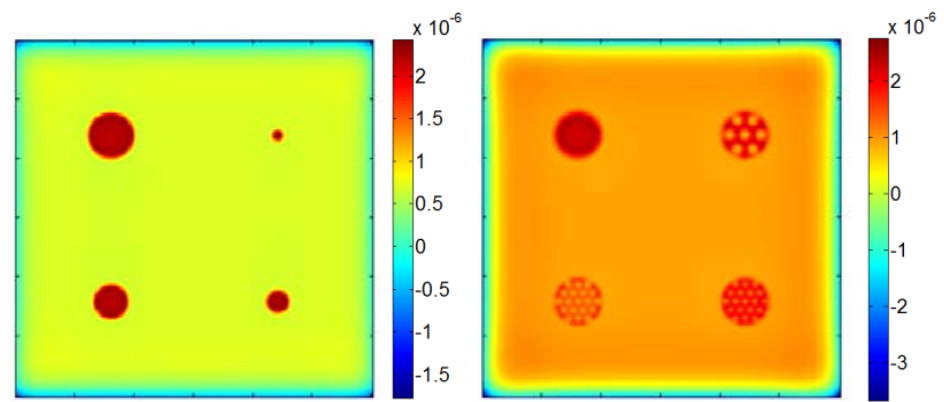

Fig. 6. Simulated out-plane-plane displacement image of the first and second sample. The images are taken at the end of the heating.

To get phase image, displacement curl has to be calculated in the shear direction and then the resulting map must be wrapped to obtain an equivalent phase map. By applying such processing to the whole displacement image sequence, it is possible to build an equivalent phase map sequence in order to test the robustness of the processing algorithms studied versus measurement noise.

\subsection{Analysis}

To interpret the results, we first look at the average displacement taken over the area covered by the defect. For example, for a $40 \mathrm{~mm}$ wide defect, the average is calculated over a 40 by $40 \mathrm{~mm}$ square centered on the defect.
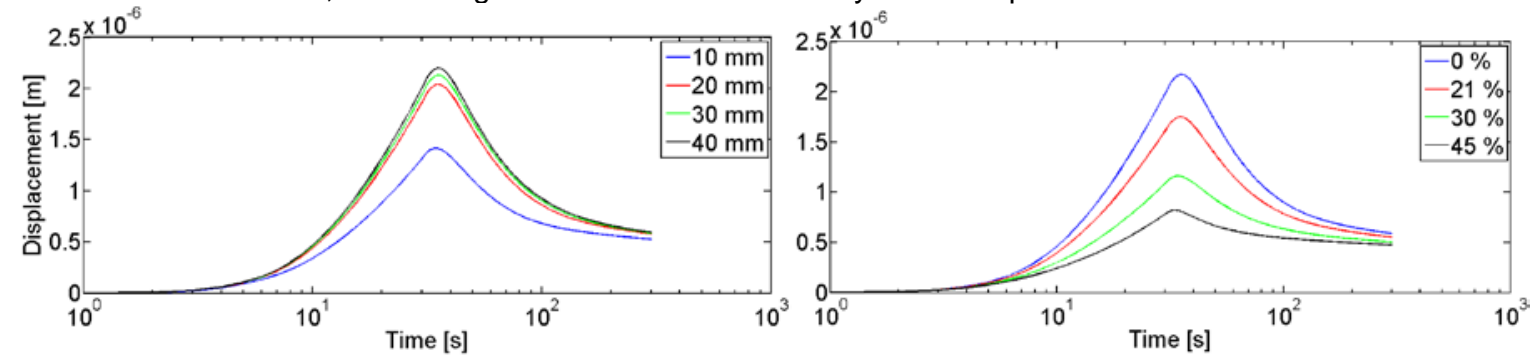

Fig. 7. Simulated out-plane-plane displacement profile of the first and second sample.

The results from the first samples show that the displacement from the defect 20,30 and $40 \mathrm{~mm}$ wide, the difference in displacement is relatively small. But for the $10 \mathrm{~mm}$ wide defect, the out-of-plane displacement is very small compared with other defect. When comparing different bonding quality in the second sample in Figure 7 , we can deduct that the displacement is inversely proportional to the bonding quality.

To understand why the $10 \mathrm{~mm}$ wide defect is different from the others, we take a closer look at the transversal shape of the surface. Figure 8 shows transversal displacement profiles for the different defect sizes. 

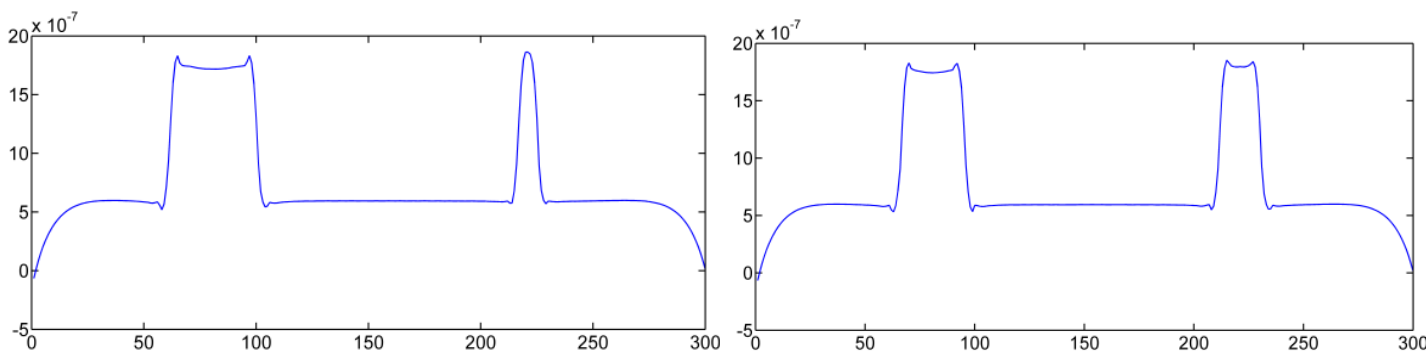

Fig.8. Transversal displacement profiles of the 40 and10 mm wide defect on the left graph and for the 30 and $20 \mathrm{~mm}$ wide defects on the right.

The shape of the out-of-plane displacement is consistent for all defects except for the smallest defect. This could explain the conflicting displacement of the $10 \mathrm{~mm}$ defect observed in the left graph of Fig. 7. We can assume that this is due to the coarse meshing of the numerical model. Perhaps refining the meshing could prevent this contradictory behavior of the small defect.

In figure 9 the temperature running contrast for both sample is presented.
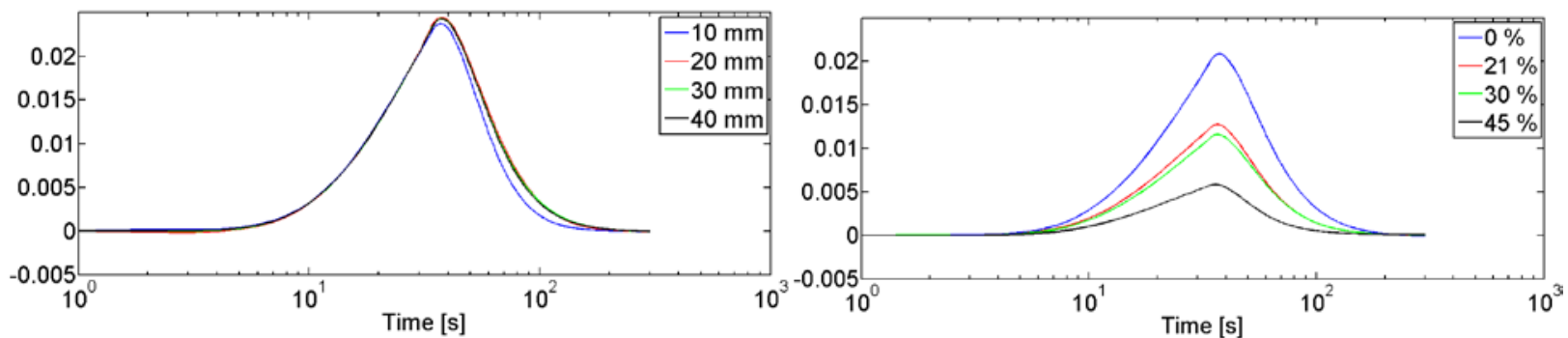

Fig. 9. Simulated temperature running contrast profile of the first and second sample.

When analysing the temperature profile, it is more relevant to look at the running contrast since the difference of temperature is very small. From these graph, it can be observed that the size of the defect doesn't seem to affect the defect temperature signature in the first sample. However, just as the displacement, the temperature running contrast is inversely proportional to the bonding quality.

Finally it is interesting to compare the evolution of the running contrast from both the displacement and temperature measurement. In figure 10 and 11 we compared running contrast calculated for displacement and temperature evolution for 2 sizes and also 2 different bonding quality of defects.
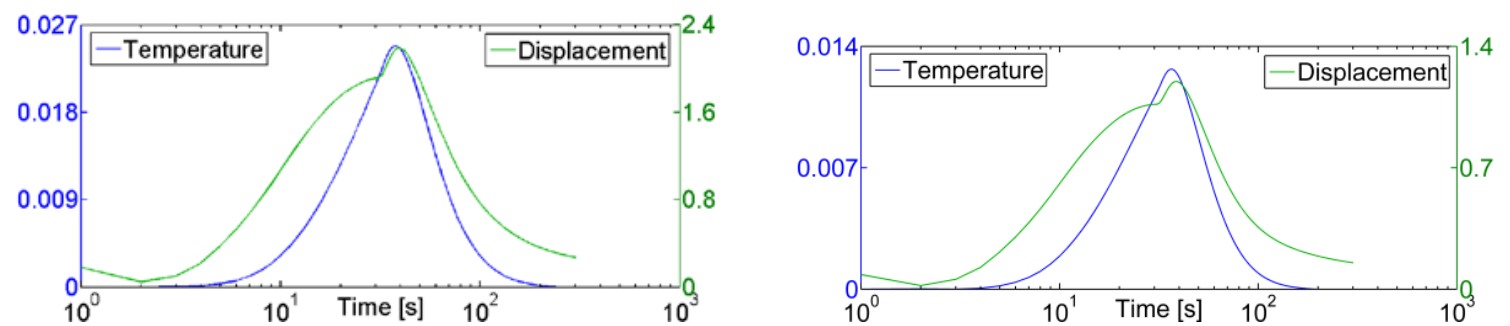

Fig 10. Running contrast for the temperature and displacement measurements for the 40 and $10 \mathrm{~mm}$ wide defects from the first sample for the left and right graph respectively.
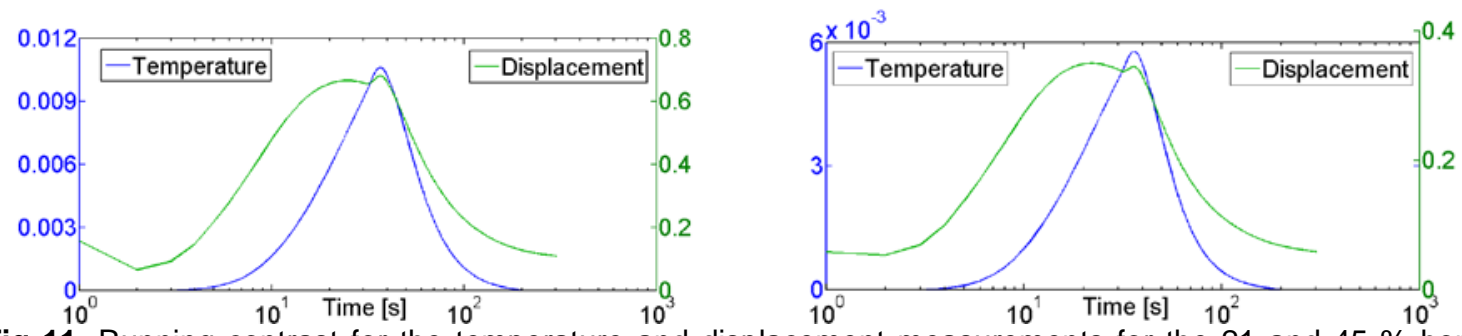

Fig 11. Running contrast for the temperature and displacement measurements for the 21 and $45 \%$ bonding quality defect in the second sample for the left and right graph respectively. 
Looking at the graphs from Fig. 10, there are similarities between both running contrasts. The time where the maximum value is relatively the same and both wave share the same shape when it reach its peak. The difference between both sizes of the defect seems to affect the difference.

From the graphs of Fig. 11, we can see that a local maximum appears for the bonding quality of $21 \%$. This maximum becomes global, when the bonding quality increases to $45 \%$. To explain this behaviour, we must first remind that two physical phenomenons are at the origin of the deformation at the surface of the sample. Displacement is caused by a thermal stress due to a temperature gradient but also due to the difference in thermal expansion in the materials. According to this remark, the first peak at 22 sec could be caused mostly by temperature difference between the CFRP and the defect. The other maximum at roughly $39 \mathrm{sec}$ could be probably caused by heat that reached the defective area interface and increase due to local thermal resistance. Since it has a different coefficient of thermal expansion than the epoxy glue and the CFRP, it caused a peak in the running contrast profile at the same time as the maximum contrast for temperature.

\section{Conclusion}

The experimentations conducted have shown that it is possible to use thermal shearography and thermography simultaneously to effectively detect flaws in CFRP bonding. Experiments in laboratory conditions also shed light on some of the limitations and possible improvements of the setup. From Figure 3, the shape of the deformation is not uniform over the area covered by the PTFE disc. We attribute this to the fact that the samples use an impregnated tissue. The uniform gluing of the tissue on the defect revealed by shearography is not visible in the thermals images.

Concerning shearography, the area of inspection was very limited due to the inhomogeneous distribution of the light beam projected. The illumination was not sufficient on the edge of the images. But when opening the diaphragm to increase the brightness, the pixels in the center become oversaturated. The diffractive lens must be chosen accordingly with the used wavelength. Another aspect of the shearographic setup that could be improved is the acquisition rate. An essential part of designing a numerical model is the validation, but it requires experimental data about the out-of-plane displacement in function of time. Currently, the experimental setup doesn't allow continuous shearographic acquisition. For the time being, since it takes four images to extracts the phase and two phase to calculate the phase difference, it take roughly 3 seconds to make a measurement. Improving the acquisition rate would results in a better understanding of the evolution in time of the thermomechanic response of the structure and would allow a more complete validation of the numerical model. This would also require that both the thermographic and the shearographic measurements are fully synchronised between each other and with the excitation source.

As to thermography, looking at the thermal images, it can be seen that the heating is mostly directed at the top of the sample. Experimental setup could be modified to improve the homogeneity of the heating and see how it impacts the measurements. Since, the lamps were positioned somewhat higher than the sample, this caused a non-uniform heating that was mostly concentrated on the top of the sample as it can be seen in the infrared thermals images. The heat excitation in the model was a uniform heat flux and should be replaced by a modeling of the lamp with a condition of surface-to-surface radiation. This will pose a serious challenge since very little information is available about the lamps, especially about the geometry of the active radiative elements.

The results extracted from the numerical simulation gave interesting insight on the relation between thermography and shearography. It was shown that both measures are affected by the bonding quality of the defect as well as its size in some extent. When looking at the evolution of the running contrast, similarities are present between the two waveforms. The time of the global maximum is the same for the temperature and displacement contrast. When increasing the bonding quality, the local maximum increases and eventually become the global maximum for the defect with the $45 \%$ bonding quality defect. This particular behaviour of the thermomechanical contrast evolution shows us the effect of the temperature gradient and the difference in thermal expansion on the thermal stress.

In the near future, we will be looking forward to make measurements on sample with glues that have different bonding strength. This will test the feasibility to use thermal shearography as a way to quantitatively evaluate the bonds strength. We also wish to test lock-in acquisition as well as a continuous phase extraction approach.

\section{Acknowledgements:}

The authors wish to thank Frederic Taillade for providing his in-house shearographic head to carry out the experimental feasibility tests. The Sense-City project (Equipex Sense-City) and NSERC (Canada) are also acknowledged. 


\section{REFERENCES}

[1] Stewart, M.G., Rosowsky D.V., Time-dependent reliability of deteriorating reinforced concrete bridge decks, Structural Safety, vol. 20, issue 1, 1998, pp. 91-109.

[2] Nanni A., North American design guidelines for concrete reinforcement and strengthening using FRP: principles, applications and unresolved issues, Construction and Building Materials, vol. 17, issue 6-7, September-October 2003, pp. 439-446.

[3] Balageas D.L., Deom A.A., Boscher D.M., Characterization and nondestructive testing of carbon-epoxy composites by a pulsed photothermal method, Journal of Materials Evaluation, vol. 45, issue 4, 1987, p. 465466.

[4] X.P.V. Maldague, Theory and practice of infrared technology for non-destructive testing, John Wiley \& sons Inc., 2001.

[5] V. Feuillet, L. Ibos, M. Fois, J. Dumoulin, Y. Candau, Defect detection and characterization in composite materials using Square Pulse Thermography coupled with Singular Value Decomposition analysis and thermal quadrupole modeling, NDT\&E International, vol. 51, Octobre 2012, pp 58-67, Elsevier, http://dx.doi.org/10.1016/j.ndteint.2012.06.003

[6] A. Crinière, J. Dumoulin, C. Ibarra-Castanedo and X. Maldague, Inverse model for defect characterization of externally glued CFRP on reinforced concrete structures: Comparative study of square pulsed and pulsed thermography, Quantitative InfraRed Thermography Journal, Taylor \& Francis Editor, 2014. DOI: 10.1080/17686733.2014.897512.

[7] J. Dumoulin, F. Taillade, Ch. Aubagnac, K. Benzarti, M. Quiertant, Infrared Thermography for the Nondestructive Inspection of CFRP Strenghtening, Concrete International, vol. 33, issue 4, April 2011, pp. 5458.

[8] Bonaldo E., Barros J.A.O., Lourenço P.B., Bond characterization between concrete substrate and repairing SFRC using pull-off testing, International Journal of Adhesion and Adhesives, vol. 25, issue 6, December 2005, pp. 463-474.

[9] Frédéric Taillade, Marc Quiertant, Karim Benzarti, Christophe Aubagnac, Shearography and pulsed stimulated infared thermography applied to a nondestructive evaluation of FRP strengthening systems bonded on concrete structures, Construction and Building Materials 25, France, 2011.

[10] Y.Y. Hung, C.Y. Liand, Image-shearing camera for direct measurement of surface strains, Applied Optics, vol.18, issue 7, 1979, pp. 1046-1051.

[11] Pascal Blain, Détection de défauts par Shearographie dans les ouvrages d'arts, Mémoire de Projet de fin d'étude, École nationale supérieur de physique de Strasbourg, France, Promotion 2007.

[12] Frédéric Taillade, Association de la shearographie et des ondes de Lamb pour la détection rapide et quantitative des délaminages, Doctorat Thesis, Conservatoire National des Arts et Métiers, 2000. 